\title{
NODAL SOLUTIONS OF WEIGHTED INDEFINITE PROBLEMS
}

\author{
M. FENCL AND J. LÓPEZ-GÓMEZ
}

This paper is dedicated to M. Hieber at the occasion of his 60th birthday

mit Wertschätzung und Freundschaft

\begin{abstract}
This paper analyzes the structure of the set of nodal solutions, i.e., solutions changing sign, of a class of one-dimensional superlinear indefinite boundary values problems with an indefinite weight functions in front of the spectral parameter. Quite surprisingly, the associated high order eigenvalues may not be concave as is the case for the lowest one. As a consequence, in many circumstances, the nodal solutions can bifurcate from three or even four bifurcation points from the trivial solution. This paper combines analytical and numerical tools. The analysis carried out is a paradigm of how mathematical analysis aids the numerical study of a problem, whereas simultaneously the numerical study confirms and illuminates the analysis.

2020 MSC: 34B15, 34B08, 34L16.

Keywords and phrases: superlinear indefinite problems, weighted problems, positive solutions, nodal solutions, eigencurves, concavity, bifurcation, global components, path-following, pseudo-spectral methods, finite-differences scheme.

Partially supported by the Research Grant PGC2018-097104-B-I00 of the Spanish Ministry of Science, Innovation and Universities, and the Institute of Inter-disciplinar Mathematics (IMI) of Complutense University. M. Fencl has been supported by the project SGS-2019-010 of the University of West Bohemia, the project 18-03253S of the Grant Agency of the Czech Republic and the project LO1506 of the Czech Ministry of Education, Youth and Sport.
\end{abstract}

\section{INTRODUCTION}

In this paper we analyze the nodal solutions of the one-dimensional nonlinear weighted boundary value problem

$$
\left\{\begin{array}{l}
-u^{\prime \prime}-\mu u=\lambda m(x) u-a(x) u^{2} \quad \text { in }(0,1), \\
u(0)=u(1)=0,
\end{array}\right.
$$

where $a, m \in \mathcal{C}[0,1]$ are functions that change sign in $(0,1)$ and $\lambda, \mu \in \mathbb{R}$ are regarded as bifurcation parameters. More precisely, $\lambda$ is the primary parameter, and $\mu$ the secondary one. All the numerical experiments carried out in this paper have been implemented in the special case when

$$
a(x):= \begin{cases}-0.2 \sin \left(\frac{\pi}{0.2}(0.2-x)\right) & \text { if } 0 \leq x \leq 0.2, \\ \sin \left(\frac{\pi}{0.6}(x-0.2)\right) & \text { if } 0.2<x \leq 0.8, \\ -0.2 \sin \left(\frac{\pi}{0.2}(x-0.8)\right) & \text { if } 0.8<x \leq 1,\end{cases}
$$


because this is the weight function $a(x)$ considered by López-Gómez and Molina-Meyer in [29] to compute the global bifurcation diagrams of positive solutions. Figure 1 shows a plot of this function. In this paper we pay a very special attention to the particular, but very interesting, case when

$$
m(x)=\sin (j \pi x)
$$

for some integer $j \geq 2$.

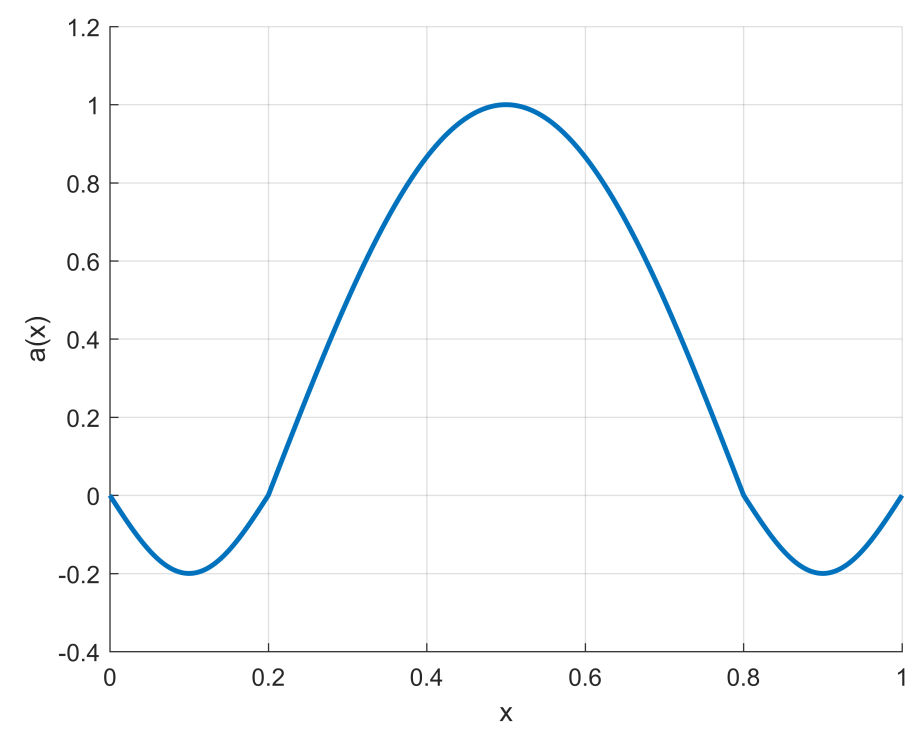

FiguRE 1. The graph of the weight function $a(x)$.

To the best of our knowledge, this is the first paper where the problem of the existence and the structure of nodal solutions of a weighted superlinear indefinite problem is addressed when $m(x)$ changes sign. The existence results for large solutions of Mawhin, Papini and Zanolin [40] required $m \equiv 1$, as well as the results of López-Gómez, Tellini and Zanolin [36], where the attention was focused on the problem of ascertaining the structure of the set of positive solutions. Most available results on nodal solutions deal with the special cases when $m \equiv 1, \mu=0$ and $a(x)$ is a positive function with $\min _{[0,1]} a>0$ (see Rabinowitz [41, 42,43]), or with the degenerate case when $a(x)$ is a continuous positive function such that $a^{-1}(0)=[\alpha, \beta] \subset(0,1)$ (see López-Gómez and Rabinowitz [37, 38, 39], and López-Gómez, Molina-Meyer and Rabinowitz [34]). In strong contrast with the classical cases when $\min _{[0,1]} a>0$, in the degenerate case when $a \geq 0$ with $a^{-1}(0)=[\alpha, \beta] \subset(0,1)$, the set of nodal solutions might consist of two, or even more, components, depending on the nature of the weight function $a(x)$ (see [34] and [39] for further details). Nevertheless, as for the special choice $a(x)$ given by $(1.2), a(x)$ is negative in the intervals $(0,0.2)$ and $(0.8,1)$, while it is positive in the central interval $(0.2,0.8)$, this is the first time that the problem of analyzing the structure of the nodal solutions in this type of superlinear indefinite problems is addressed. 
A natural strategy for constructing the solutions of (1.1) with $n \geq 0$ interior zeroes, or nodes, consists in linearizing (1.1) at the trivial solution, $u=0$, and then searching for the eigenvalues of the linearization having an associated eigenfunction with exactly $n$ interior nodes in $(0,1)$, for as these values of the parameters will provide us, through the local bifurcation theorem of Crandall and Rabinowitz [12], with all the small nodal solutions of (1.1) bifurcating from $u=0$. This strategy leads us, in a rather natural way, to the linear weighted eigenvalue problem

$$
\left\{\begin{array}{l}
-\varphi^{\prime \prime}-\mu \varphi-\lambda m(x) \varphi=\sigma \varphi \quad \text { in }(0,1), \\
\varphi(0)=\varphi(1)=0 .
\end{array}\right.
$$

By Sturm-Liouville theory, Problem (1.3) has a sequence of eigenvalues

$$
\Sigma_{n}(\lambda, \mu):=\sigma_{n}\left[-D^{2}-\mu-\lambda m(x) ;(0,1)\right], \quad n \geq 1,
$$

which are algebraically simple. Moreover, associated with each of them there is an eigenfunction, $\varphi_{n}$, with $\varphi_{n}^{\prime}(0)>0$, unique up to a multiplicative constant, with exactly $n-1$ interior nodes, necessarily simple, in $(0,1)$. By uniqueness,

$$
\Sigma_{n}(\lambda, \mu):=\sigma_{n}\left[-D^{2}-\lambda m(x) ;(0,1)\right]-\mu, \quad n \geq 1 .
$$

It turns out that the set of all the possible bifurcation points from $u=0$ to solutions of (1.1) with $n-1$ interior zeroes corresponds to values of $\lambda$ and $\mu$ for which

$$
\Sigma_{n}(\lambda, \mu)=0 .
$$

This motivates the analysis of these curves. Throughout this paper, we will denote

$$
\Sigma_{n}(\lambda):=\Sigma(\lambda, 0)=\sigma_{n}\left[-D^{2}-\lambda m(x) ;(0,1)\right], \quad n \geq 1 .
$$

Then,

$$
\Sigma_{n}(\lambda, \mu)=\Sigma_{n}(\lambda)-\mu
$$

and $\Sigma_{n}(0)=(n \pi)^{2}$ for all $n \geq 1$. Based on a classical result of Kato [22] on perturbation of simple eigenvalues, for every $n \geq 1, \Sigma_{n}(\lambda)$ is analytic in $\lambda \in \mathbb{R}$. A proof of this can be obtained by means of [27, Ch. 9] and Section 5 of Antón and López-Gómez [2], where the result was established when $n=1$. An extremely important property of $\Sigma_{1}(\lambda)$ is its strict concavity with respect to the parameter $\lambda$ (see Berestycki, Nirenberg and Varadhan [5], Cano-Casanova and López-Gómez [10] and Chapter 9 of [27]). It holds that $\Sigma_{1}^{\prime}(\lambda)>0$ for all $\lambda<0, \Sigma_{1}^{\prime}(0)=0, \Sigma_{1}^{\prime}(\lambda)<0$ for all $\lambda>0$, and

$$
\Sigma_{1}^{\prime \prime}(\lambda)<0 \text { for all } \lambda \in \mathbb{R} \text {. }
$$

Since $\Sigma_{1}(0)=\pi^{2}$, this property entails that, for every $\mu<\pi^{2}, \Sigma_{1}^{-1}(\mu)$ consists of two values of $\lambda$,

$$
\lambda_{-} \equiv \lambda_{-}(\mu)<0<\lambda_{+} \equiv \lambda_{+}(\mu),
$$

which are the unique bifurcation values to positive solutions from $u=0$ of (1.1) (see López-Gómez and Molina-Meyer [29]). Even dealing with general second order elliptic operators under general mixed boundary conditions of non-classical type, the strict concavity of $\Sigma_{1}(\lambda)$ relies on the strong ellipticity of the operator (see, e.g., Chapter 8 of $[27])$. 
For analytic semigroups the spectral mapping theorem holds (see, e.g., [3, 4]), i.e,

$$
\sigma\left(e^{D^{2}+\lambda m}\right) \backslash\{0\}=e^{-\sigma\left(-D^{2}-\lambda m\right)}=\left\{e^{-\sigma_{n}\left(-D^{2}-\lambda m ;(0,1)\right)}: n \geq 1\right\} .
$$

Thus, the spectral radius of the associated semigroup is given through the formula

$$
\varrho(\lambda):=\operatorname{spr}\left(e^{D^{2}+\lambda m}\right)=e^{-\sigma_{1}\left(-D^{2}-\lambda m ;(0,1)\right)}=e^{-\Sigma_{1}(\lambda)}, \quad \lambda \in \mathbb{R} .
$$

Hence, $\varrho(\lambda)$ is logarithmically convex, which is a classical property observed by Kato [21], and equivalent to the concavity of $\Sigma_{1}(\lambda)$.

According to Propositions 1.10 and 1.11 of Figueiredo [17], for every $\mu \leq 0$, the eigenvalue problem

$$
\left\{\begin{array}{l}
-\varphi^{\prime \prime}-\mu \varphi=\lambda m(x) \varphi \quad \text { in }(0,1) \\
\varphi(0)=\varphi(1)=0
\end{array}\right.
$$

has a double sequence of eigenvalues

$$
\cdots \leq \lambda_{-2}(\mu) \leq \lambda_{-1}(\mu)<0<\lambda_{1}(\mu) \leq \lambda_{2}(\mu) \leq \cdots
$$

such that $\lim _{n \rightarrow \infty} \lambda_{n}(\mu)=+\infty$ and $\lim _{n \rightarrow \infty} \lambda_{-n}(\mu)=-\infty$. Actually, by a result of Hess and Kato [20], $\lambda_{-2}(\mu)<\lambda_{-1}(\mu)<0<\lambda_{1}(\mu)<\lambda_{2}(\mu)$. Thus, it is rather natural to believe that in the one-dimensional setting $\lambda_{-(n+1)}(\mu)<\lambda_{-n}(\mu)$ and $\lambda_{n}(\mu)<$ $\lambda_{n+1}(\mu)$ for all $n \geq 1$, and that, for every $n \in \mathbb{Z}$, associated to $\lambda_{n}(\mu)$ there is a unique eigenfunction, up to a multiplicative constant, $\varphi_{n}$, with $n-1$ interior nodes in $(0,1)$, as it occurs in the setting of the Sturm-Liouville theory. Rather surprisingly, this is not true for sufficiently large $\mu>0$ because $\Sigma_{n}(\lambda)$ is not necessarily concave for all $n \geq 2$. Actually, there are examples of weight functions $m(x)$ for which none of the remaining eigenvalues $\Sigma_{n}(\lambda), n \geq 2$, is concave with respect to $\lambda$. Figure 2 shows one of these examples for the special choice $m(x)=\sin (2 \pi x)$. In this case, $\Sigma_{1}(\lambda)$ is the unique eigencurve which is concave. All other curves are symmetric functions of $\lambda$, with a quadratic local minimum at $\lambda=0$, as illustrated by Figure 2. This fact has dramatic implications from the point of view of the structure of the set of nodal solutions of the problem (1.1). Indeed, setting

$$
\mu_{n}=\max _{\lambda \in \mathbb{R}} \Sigma_{n}(\lambda), \quad n \geq 1,
$$

it is easily seen that $\mu_{n}>\Sigma_{n}(0)=(n \pi)^{2}$ for all $n \geq 1$ and, hence, for every $n \geq 2$ and any $\mu \in\left((n \pi)^{2}, \mu_{n}\right), \Sigma_{n}^{-1}(\mu)$ consists of two negative eigenvalues, $\lambda_{-[1, n]}(\mu)<$ $\lambda_{-[2, n]}(\mu)<0$, and two positive eigenvalues $0<\lambda_{+[2, n]}(\mu)<\lambda_{+[1, n]}(\mu)$ such that

$$
0<\lambda_{+[2, n]}(\mu)=-\lambda_{-[2, n]}(\mu)<\lambda_{+[1, n]}(\mu)=-\lambda_{-[1, n]}(\mu) .
$$

Therefore, for this range of $\mu$ 's we expect that the solutions with $n-1$ interior nodes of (1.1) will bifurcate from the trivial solution at each of the four values

$$
\lambda=\lambda_{ \pm[i, n]}, \quad i=1,2 .
$$

By construction, letting $\mu \downarrow(n \pi)^{2}$, it follows that

$$
\lambda_{ \pm[2, n]}\left((n \pi)^{2}\right)=0 .
$$

Moreover,

$$
\lambda_{-[1, n]}\left(\mu_{n}\right)=\lambda_{-[2, n]}\left(\mu_{n}\right)<0<\lambda_{+[2, n]}\left(\mu_{n}\right)=\lambda_{+[1, n]}\left(\mu_{n}\right),
$$

at least for $n \in\{2,3,4,5\}$. 


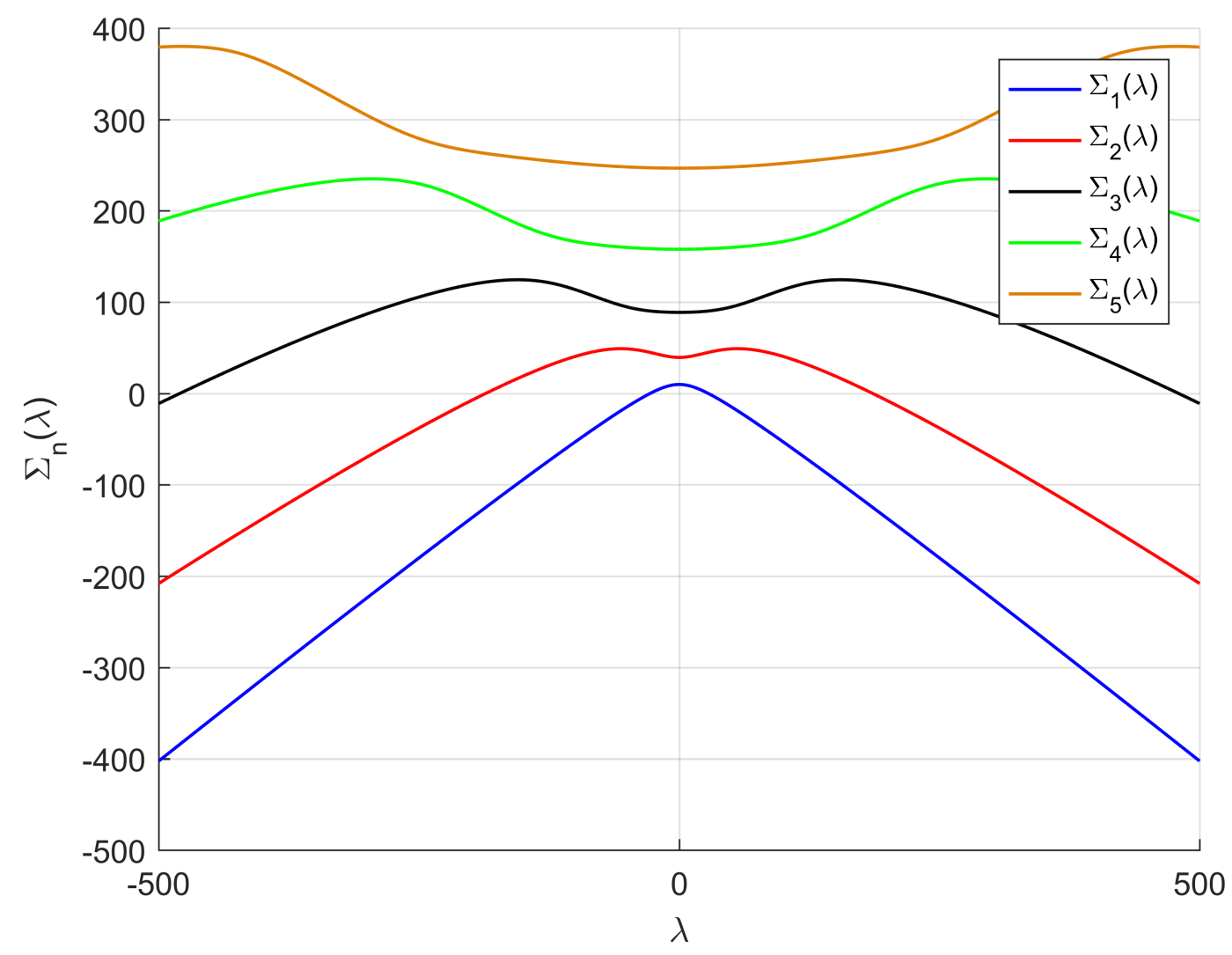

Figure 2. The curves $\Sigma_{n}(\lambda)$ for $1 \leq n \leq 5$ and $m(x)=\sin (2 \pi x)$.

As illustrated in Figure 3, the number of eigencurves, $\Sigma_{n}(\lambda), n \geq 2$, which are concave in $\lambda$ might vary with the weight function $m(x)$. Indeed, when $m(x)=\sin (4 \pi x)$, it turns out that not only $\Sigma_{1}(\lambda)$ but also $\Sigma_{2}(\lambda)$ is strictly concave, while the remaining eigencurves, $\Sigma_{n}(\lambda)$, with $n \geq 3$, are not concave. Similarly, when $m(x)=\sin (6 \pi x)$, then $\Sigma_{j}(\lambda)$ are concave for $j \in\{1,2,3\}$, while they are not concave for $j \geq 4$.

As suggested by our numerical computations, the more wiggled $m(x)$ is, the higher the number of modes is for which $\Sigma_{n}(\lambda)$ is concave.

The structure of this paper is as follows. Section 2 studies some global properties of the eigencurves $\Sigma_{n}(\lambda)$ for all $n \geq 2$ and analyzes their concavities in the special case when, for some $k \geq 1$,

$$
m(x)=\sin (2 k \pi x), \quad x \in[0,1] .
$$

In Section 3 some global bifurcation diagrams of nodal solutions of (1.1) with one and two interior nodes are derived; they complement the global bifurcation diagrams of positive solutions of López-Gómez and Molina-Meyer [29]. Finally, in Section 4 we describe the numerical schemes used to get the global bifurcation diagrams of Section 3 . 

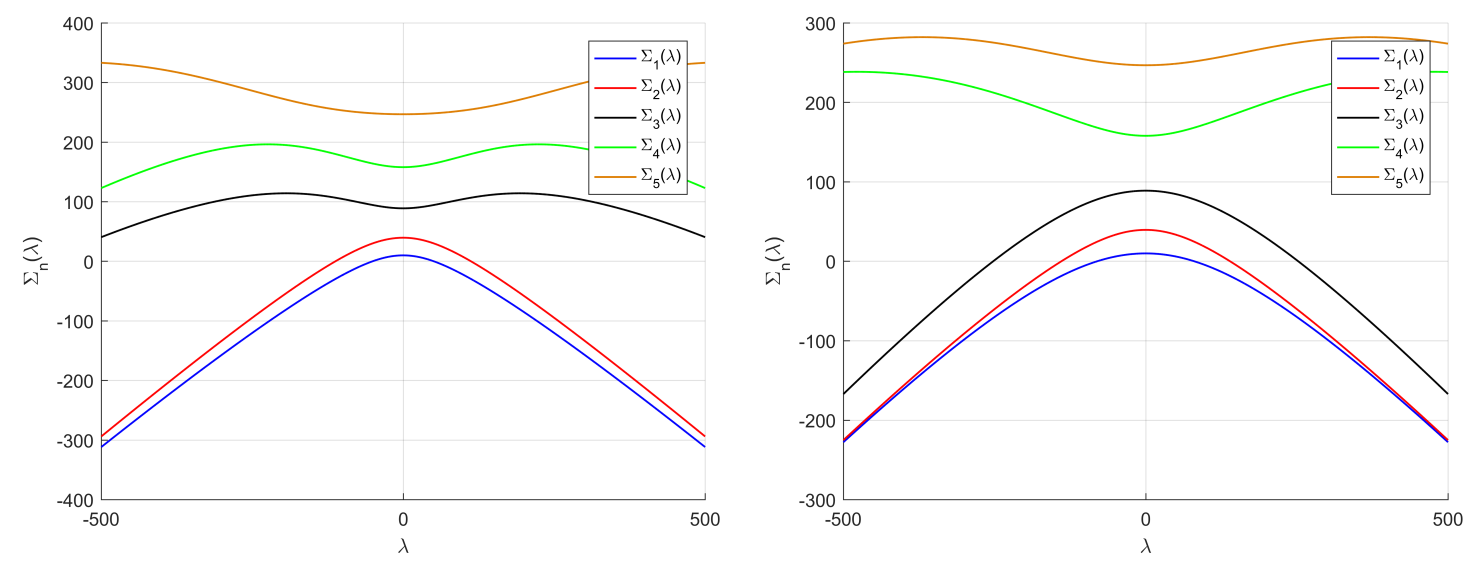

FiguRE 3. The curves $\Sigma_{n}(\lambda)$ for $1 \leq n \leq 5$ with $m(x)=\sin (j \pi x), j=4,6$.

\section{Some Global properties of the nodal eigencurves $\Sigma_{n}(\lambda)$}

Throughout this paper, for any given $r, s \in \mathbb{R}$ with $r<s$ and every continuous function $q \in \mathcal{C}[r, s]$, we denote by $\sigma_{n}\left[-D^{2}+q(x) ;(r, s)\right], n \geq 1$, the $n$-th eigenvalue of the eigenvalue problem

$$
\left\{\begin{array}{l}
-\varphi^{\prime \prime}+q(x) \varphi=\sigma \varphi \quad \text { in }(r, s), \\
\varphi(r)=\varphi(s)=0 .
\end{array}\right.
$$

The next properties are well known (see, e.g., [9]):

i) Monotonicity of $\sigma_{n}$ with respect to $q(x)$ : If $q, \tilde{q} \in \mathcal{C}[r, s]$ satisfy $q \lesseqgtr \tilde{q}$, then

$$
\sigma_{n}\left[-D^{2}+q(x) ;(r, s)\right]<\sigma_{n}\left[-D^{2}+\tilde{q} ;(r, s)\right] \text { for all } n \geq 1 .
$$

ii) Monotonicity of $\sigma_{n}$ with respect to the interval: If $[\alpha, \beta] \subset(r, s)$, then

$$
\sigma_{n}\left[-D^{2}+q ;(r, s)\right]<\sigma_{n}\left[-D^{2}+q ;(\alpha, \beta)\right] \text { for all } n \geq 1 \text {. }
$$

Based on these properties, as suggested by Figures 2 and 3, the next result holds.

Proposition 2.1. Suppose that there exist $x_{ \pm} \in(0,1)$ such that $\pm m\left(x_{ \pm}\right)>0$, i.e., $m(x)$ changes the sign in $(0,1)$. Then, for every $n \geq 1$,

$$
\lim _{\lambda \downarrow-\infty} \Sigma_{n}(\lambda)=-\infty, \quad \lim _{\lambda \uparrow \infty} \Sigma_{n}(\lambda)=-\infty .
$$

Proof. Consider a sufficiently small $\varepsilon>0$ such that

$$
J_{\varepsilon}:=\left[x_{+}-\varepsilon, x_{+}+\varepsilon\right] \subset(0,1), \quad \min _{J_{\varepsilon}} m=m_{L}>0 .
$$

Then, by the monotonicity properties of $\Sigma_{n}$, for every $\lambda>0$ and $n \geq 1$, we have that

$$
\begin{aligned}
\Sigma_{n}(\lambda) & =\sigma_{n}\left[-D^{2}-\lambda m(x) ;(0,1)\right]<\sigma_{n}\left[-D^{2}-\lambda m(x) ; J_{\varepsilon}\right] \\
& <\sigma_{n}\left[-D^{2}-\lambda m_{L} ; J_{\varepsilon}\right]=\sigma_{n}\left[-D^{2} ; J_{\varepsilon}\right]-\lambda m_{L}=\left(\frac{n \pi}{2 \varepsilon}\right)^{2}-\lambda m_{L} .
\end{aligned}
$$

Thus, letting $\lambda \uparrow \infty$, the second relation of (2.2) holds. The first one follows by applying this result to the weight function $-m(x)$. 
The fact that all the eigencurves plotted in Figures 2 and 3 are symmetric about the $y$-axis is a direct consequence of the next general result, because

$$
\sin (2 k \pi(1-x))=-\sin (2 k \pi x),
$$

for all integer $k \geq 1$ and $x \in[0,1]$.

Proposition 2.2. Suppose that $m \neq 0$ is a continuous function in $[0,1]$ such that

$$
m(1-x)=-m(x) \quad \text { for all } x \in[0,1] ;
$$

this holds assuming (1.8). Then, $\Sigma_{n}(-\lambda)=\Sigma_{n}(\lambda)$ for all $\lambda \in \mathbb{R}$ and any integer $n \geq 1$. In particular,

$$
\dot{\Sigma}_{n}(0)=0 \text { for all } n \geq 1,
$$

where we are denoting $\dot{\Sigma}_{n}=\frac{d \Sigma_{n}}{d \lambda}$.

Proof. Since $m \neq 0$, either there exists $x_{+} \in(0,1)$ such that $m\left(x_{+}\right)>0$, or $m\left(x_{-}\right)<0$ for some $x_{-} \in(0,1)$. Suppose the first alternative occurs. Then, by $(2.3)$, we also have that

$$
m\left(1-x_{+}\right)=-m\left(x_{+}\right)<0
$$

and hence, $m(x)$ changes the sign in $(0,1)$. In particular, (2.2) holds.

Pick an integer $n \geq 1$, a real number $\lambda$, and let $\phi_{n}$ be an eigenfunction associated to $\Sigma_{n}(\lambda)$. Then, $\phi_{n}$ possesses $n-1$ zeros in $(0,1), \phi_{n}(0)=\phi_{n}(1)=0$, and

$$
-\phi_{n}^{\prime \prime}(x)=\lambda m(x) \phi_{n}(x)+\Sigma_{n}(\lambda) \phi_{n}(x)
$$

for all $x \in(0,1)$. Thus, setting

$$
\psi_{n}(x):=\phi_{n}(1-x), \quad x \in[0,1]
$$

it is easily seen that

$$
\psi_{n}^{\prime}(x):=-\phi_{n}^{\prime}(1-x), \quad \psi_{n}^{\prime \prime}(x)=\phi_{n}^{\prime \prime}(1-x), \quad x \in[0,1],
$$

and hence, for every $x \in(0,1)$,

$$
\begin{aligned}
-\psi_{n}^{\prime \prime}(x)=-\phi_{n}^{\prime \prime}(1-x) & =\lambda m(1-x) \phi_{n}(1-x)+\Sigma_{n}(\lambda) \phi_{n}(1-x) \\
& =\lambda m(1-x) \psi_{n}(x)+\Sigma_{n}(\lambda) \psi_{n}(x) \\
& =-\lambda m(x) \psi_{n}(x)+\Sigma_{n}(\lambda) \psi_{n}(x) .
\end{aligned}
$$

Consequently, $\psi_{n}(x)$ is an eigenfunction associated to $-D^{2}+\lambda m(x)$ with $n-1$ interior zeros. Therefore, by the uniqueness of $\Sigma_{n}$, it becomes apparent that

$$
\Sigma_{n}(-\lambda)=\Sigma_{n}(\lambda) \text { for all } \lambda \in \mathbb{R} .
$$

Since $\Sigma_{n}(\lambda)$ is an analytic function of $\lambda$, necessarily $\dot{\Sigma}_{n}(0)=0$.

Figure 4 shows that the function $\Sigma_{n}(\lambda)$ may not be an even function of $\lambda$ if condition (2.3) fails.

The next result establishes that, as already suggested by Figures 2 and 3, the nodal eigencurves, $\Sigma_{n}(\lambda)$, cannot be concave for the choice (1.8) if $n \geq k+1$. We conjecture that, in general, for that particular choice, $\Sigma_{n}$ is concave if $n \leq k$. Therefore, $\Sigma_{n}$ should be concave if, and only if, $n \leq k$. The analysis of the concavity when $n \leq k$ for the choice (1.8) remains outside the general scope of this paper. 

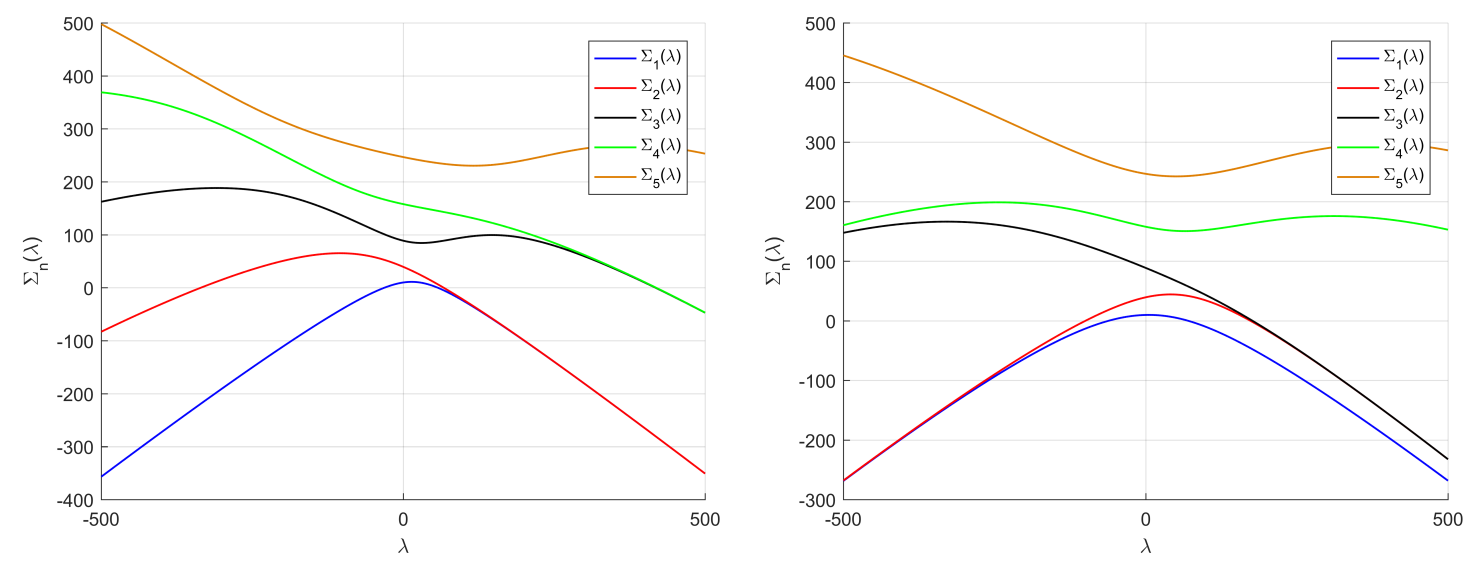

Figure 4 . The curves $\Sigma_{n}(\lambda)$ for $1 \leq n \leq 5$ with $m(x)=\sin (j \pi x), j=3,5$.

Theorem 2.1. Assume (1.8) for some integer $k \geq 1$. Then, as soon as $n \geq k+1$,

$$
\ddot{\Sigma}_{n}(0)>0 \quad \text { for all } n \geq k+1 \text {. }
$$

Therefore, by (2.4), $\lambda=0$ is a local minimum of $\Sigma_{n}(\lambda)$ and, in particular, $\Sigma_{n}(\lambda)$ cannot be concave.

Proof. Since $\Sigma_{n}(\lambda)$ is algebraically simple for all $n \geq 1$, we already know that $\Sigma_{n}(\lambda)$ is analytic, by well-known perturbation results of Kato [21]. Moreover, the eigenfunction associated to $\Sigma_{n}(\lambda)$, denoted by $\varphi_{[n, \lambda]}$, can be chosen to be analytic in $\lambda$ by normalizing it so that

$$
\int_{0}^{1} \varphi_{[n, \lambda]}^{2}(x) d x=\frac{1}{2} .
$$

By definition, $\varphi_{[n, \lambda]}(0)=\varphi_{[n, \lambda]}(1)=0$ and

$$
-\varphi_{[n, \lambda]}^{\prime \prime}(x)=\lambda m(x) \varphi_{[n, \lambda]}(x)+\Sigma_{n}(\lambda) \varphi_{[n, \lambda]}(x) \text { for all } x \in(0,1) .
$$

Thus, since $\Sigma_{n}(0)=(n \pi)^{2}$, evaluating (2.7) at $\lambda=0$ and taking (2.6) into account, it becomes apparent that $\varphi_{[n, \lambda]}$ is an analytic perturbation of the eigenfunction

$$
\varphi_{[n, 0]}(x)=\sin (n \pi x), \quad x \in[0,1] .
$$

Moreover, differentiating (2.7) with respect to $\lambda$ yields

$$
-\dot{\varphi}_{[n, \lambda]}^{\prime \prime}(x)=\lambda m \dot{\varphi}_{[n, \lambda]}+m \varphi_{[n, \lambda]}+\dot{\Sigma}_{n}(\lambda) \varphi_{[n, \lambda]}+\Sigma_{n}(\lambda) \dot{\varphi}_{[n, \lambda]} \quad \text { in }(0,1) .
$$

Thus, since $\Sigma_{n}(0)=(n \pi)^{2}$ and $\dot{\Sigma}_{n}(0)=0$, evaluating (2.8) at $\lambda=0$ shows that $\dot{\varphi}_{[n, 0]}$ solves the problem

$$
\left\{\begin{array}{l}
{\left[-D^{2}-(n \pi)^{2}\right] u=m \varphi_{[n, 0]} \quad \text { in }(0,1),} \\
u(0)=u(1)=0
\end{array}\right.
$$

In order to find $\dot{\varphi}_{[n, 0]}$, we first determine the general solution of the linear inhomogeneous equation

$$
\left[-D^{2}-(n \pi)^{2}\right] u=m(x) \sin (n \pi x)
$$


Set $v:=u^{\prime}$ in order to vary coefficients in the first order system associated to (2.10),

$$
\left(\begin{array}{l}
u^{\prime} \\
v^{\prime}
\end{array}\right)=\left(\begin{array}{cc}
0 & 1 \\
-(n \pi)^{2} & 0
\end{array}\right)\left(\begin{array}{l}
u \\
v
\end{array}\right)+\left(\begin{array}{c}
0 \\
-m(x) \sin (n \pi x)
\end{array}\right) .
$$

Since

$$
W(x):=\left(\begin{array}{cc}
\cos (n \pi x) & \sin (n \pi x) \\
-n \pi \sin (n \pi x) & n \pi \cos (n \pi x)
\end{array}\right)
$$

is a fundamental matrix of solutions for the homogeneous linear system associated to (2.11), the change of variable

$$
\left(\begin{array}{l}
u \\
v
\end{array}\right)=W(x)\left(\begin{array}{l}
c_{1}(x) \\
c_{2}(x)
\end{array}\right)
$$

transforms (2.11) into the equivalent system

$$
W(x)\left(\begin{array}{c}
c_{1}^{\prime}(x) \\
c_{2}^{\prime}(x)
\end{array}\right)=\left(\begin{array}{c}
0 \\
-m(x) \sin (n \pi x)
\end{array}\right),
$$

whose solution, according to Cramer's rule, is given through

$$
c_{1}^{\prime}(x)=\frac{1}{n \pi} m(x) \sin ^{2}(n \pi x), \quad c_{2}^{\prime}(x)=\frac{-1}{n \pi} m(x) \sin (n \pi x) \cos (n \pi x) .
$$

Thus,

$$
\begin{aligned}
& c_{1}(x)=\frac{1}{n \pi} \int_{0}^{x} m(s) \sin ^{2}(n \pi s) d s+A, \\
& c_{2}(x)=-\frac{1}{n \pi} \int_{0}^{x} m(s) \sin (n \pi s) \cos (n \pi s) d s+B,
\end{aligned}
$$

for some constants $A, B \in \mathbb{R}$. Therefore, the general solution of (2.10) is given by

$$
\begin{aligned}
u(x)= & \cos (n \pi x) c_{1}(x)+\sin (n \pi x) c_{2}(x) \\
= & \cos (n \pi x)\left(A+\frac{1}{n \pi} \int_{0}^{x} m(s) \sin ^{2}(n \pi s) d s\right) \\
& +\sin (n \pi x)\left(B-\frac{1}{n \pi} \int_{0}^{x} m(s) \sin (n \pi s) \cos (n \pi s) d s\right) \\
= & A \cos (n \pi x)+B \sin (n \pi x)+p(x),
\end{aligned}
$$

where

$$
p(x):=\frac{1}{n \pi} \int_{0}^{x} m(s) \sin (n \pi s) \sin [n \pi(s-x)] d s, \quad x \in[0,1]
$$


is a particular solution of (2.10). It is the solution obtained by making the choice $A=B=0$. Obviously, $p(0)=0$. Moreover, by $(1.8)$,

$$
\begin{aligned}
p(1) & =\int_{0}^{1} m(s) \sin (n \pi s) \sin [n \pi(s-1)] d s \\
& =(-1)^{n} \int_{0}^{1} \sin (2 k \pi s) \sin ^{2}(n \pi s) d s=0,
\end{aligned}
$$

because the integrand,

$$
\theta(s):=\sin (2 k \pi s) \sin ^{2}(n \pi s), \quad s \in[0,1],
$$

satisfies $\theta(1-s)=-\theta(s)$ for all $s \in[0,1]$ and hence, it is odd about 0.5. As we are interested in solving (2.9), we should make the choice

$$
0=u(0)=A+p(0)=A \text {. }
$$

Thus,

$$
\dot{\varphi}_{[n, 0]}(x)=B \sin (n \pi x)+p(x), \quad x \in[0,1],
$$

for some constant $B \in \mathbb{R}$. To determine $B$, we can proceed as follows. Differentiating (2.6) with respect to $\lambda$ and evaluating the resulting identity at $\lambda=0$ yields

$$
0=\int_{0}^{1} \varphi_{[n, 0]}(x) \dot{\varphi}_{[n, 0]}(x) d x=B \int_{0}^{1} \sin ^{2}(n \pi x) d x+\int_{0}^{1} \sin (n \pi x) p(x) d x .
$$

Consequently,

$$
B=-2 \int_{0}^{1} \sin (n \pi x) p(x) d x
$$

and therefore,

$$
\dot{\varphi}_{[n, 0]}(x)=-2\left(\int_{0}^{1} \sin (n \pi s) p(s) d s\right) \sin (n \pi x)+p(x), \quad x \in[0,1] .
$$

To find $\ddot{\Sigma}_{n}(0)$, we can differentiate identity (2.8) with respect to $\lambda$. After rearranging terms, this gives

$$
\left[-D^{2}-\lambda m-\Sigma_{n}(\lambda)\right] \ddot{\varphi}_{[n, \lambda]}=2 m \dot{\varphi}_{[n, \lambda]}+2 \dot{\Sigma}_{n}(\lambda) \dot{\varphi}_{[n, \lambda]}+\ddot{\Sigma}_{n}(\lambda) \varphi_{[n, \lambda]} .
$$

Thus, evaluating at $\lambda=0$ yields

$$
\left[-D^{2}-(n \pi)^{2}\right] \ddot{\varphi}_{[n, 0]}=2 m \dot{\varphi}_{[n, 0]}+\ddot{\Sigma}_{n}(0) \varphi_{[n, 0]}
$$

and hence, multiplying $(2.14)$ by $\varphi_{[n, 0]}$ and integrating over $(0,1)$, it is apparent that

$$
\ddot{\Sigma}_{n}(0)=-4 \int_{0}^{1} m(x) \dot{\varphi}_{[n, 0]}(x) \varphi_{[n, 0]}(x) d x .
$$

Therefore, substituting (2.13) into (2.15) and using (1.8) yields

$$
\begin{aligned}
\ddot{\Sigma}_{n}(0) & =-4 \int_{0}^{1} m(x) \varphi_{[n, 0]}(x) p(x) d x \\
& =-4 \int_{0}^{1} \sin (2 k \pi x) \sin (n \pi x)\left[\frac{1}{n \pi} \int_{0}^{x} \sin (2 k \pi s) \sin (n \pi s) \sin (n \pi(s-x)) d s\right] d x .
\end{aligned}
$$


Finally, we need the trigonometric formulas

$$
\begin{aligned}
\sin x \sin y & =\frac{1}{2}[\cos (x-y)-\cos (x+y)], \\
\sin x \cos y & =\frac{1}{2}[\sin (x-y)+\sin (x+y)],
\end{aligned}
$$

to simplify the integrands in $\ddot{\Sigma}_{n}(0)$. First, we will determine the function $p(x)$. For this, we use formula (2.16) on $\sin (2 k \pi s) \sin (n \pi s)$ and then formula (2.17) to simplify the integrand in $p(x)$. Then, integrating yields

$$
p(x)=-\frac{1}{8 \pi^{2}}\left[\frac{\cos (\pi x(2 k-n))}{k(n-k)}+\frac{\cos (\pi x(2 k+n))}{k(n+k)}-\frac{n \cos (n \pi x)}{k\left(n^{2}-k^{2}\right)}\right] .
$$

After substituting (2.18) into the formula for $\ddot{\Sigma}_{n}(0)$, we can again use formulae (2.16) and (2.17) to simplify the underlying integrands, which can then be directly integrated. The result can be simplified to get the final formula

$$
\ddot{\Sigma}_{n}(0)=\frac{1}{4 \pi^{2}\left(n^{2}-k^{2}\right)} .
$$

Obviously, $n^{2}-k^{2}>0$ if $n \geq k+1$, and therefore $\ddot{\Sigma}_{n}(0)>0$. Hence, the eigencurves $\Sigma_{n}(\lambda)$ are convex in a neighborhood of $\lambda=0$ for $n \geq k+1$. Thus, they cannot be globally concave.

\section{Global Bifurcation of nOdal solutions}

Since $\Sigma_{1}(0)=\pi^{2}$, for every $\mu<\pi^{2}$ the set $\Sigma_{1}^{-1}(\mu)$ consists of two points,

$$
\lambda_{-}(\mu)<0<\lambda_{+}(\mu) \text {, }
$$

such that

$$
\lim _{\mu \uparrow \pi^{2}} \lambda_{ \pm}(\mu)=0 .
$$

Moreover, owing to Theorem 9.4 of [27],

$$
\dot{\Sigma}_{1}\left(\lambda_{-}(\mu)\right)>0 \text { and } \dot{\Sigma}_{1}\left(\lambda_{+}(\mu)\right)<0 .
$$

Thus, by the main theorem of Crandall and Rabinowitz [12] (one can see also Chapter 2 of [26]), $\lambda=\lambda_{ \pm}(\mu)$ are the unique bifurcation values of $\lambda$ to positive solutions of (1.1) from $u=0$. The first plot of Figure 1 of López-Gómez and Molina-Meyer [29] shows one of those bifurcation diagrams for the special choice (1.2) of $a(x)$ with

$$
m(x)=\sin (2 \pi x), \quad x \in[0,1] .
$$

In order to complement the numerical experiments of [29] with our new findings here, all the numerical experiments of this section have been carried out for this special choice of $m(x)$. As $\mu$ increases to reach the critical value $\mu=\pi^{2}$, the set of positive solutions of (1.1) bifurcating from $u=0$ consists of one single closed loop bifurcating from $u=0$ at the single point $\lambda=0$. These loops, separated from $u=0$, are persistent for a large range of values of $\mu>\pi^{2}$, until they shrink to a single point before disappearing at some critical value of the parameter $\mu$ (see [29, Fig. 1]).

According to Theorem 2.1, $\Sigma_{2}(\lambda)$ is not concave if (3.1) holds, which is clearly illustrated by simply looking at the plot of $\Sigma_{2}(\lambda)$ in Figure 2 . This feature has important implications concerning the structure of the set of 1-node solutions of (1.1). Indeed, 
according to the plot of $\Sigma_{2}(\lambda)$, for every $\mu<(2 \pi)^{2}$, the set $\Sigma_{2}^{-1}(\mu)$ consists of two single values $\lambda_{-}(\mu)<0<\lambda_{+}(\mu)$ with $\dot{\Sigma}_{2}\left(\lambda_{-}(\mu)\right)>0$ and $\dot{\Sigma}_{2}\left(\lambda_{+}(\mu)\right)<0$. Thus, according to [27, Th. 9.4], the transversality condition of Crandall and Rabinowitz [12] holds at $(\lambda, u)=\left(\lambda_{ \pm}(\mu), 0\right)$. Hence, an analytic curve of 1-node solutions of (1.1) emanates from $u=0$ at each of these values of $\lambda, \lambda_{ \pm}(\mu)$. Figure 5 a shows the plots of these two curves for the value of the parameter $\mu=35$. Our numerical experiments suggest that they are separated from each other. In this bifurcation diagram, as well as in all the remaining ones, we are plotting the values of $\lambda$ versus the $L^{2}$-norm of the solutions. So, each point on the curves of the bifurcation diagrams, $(\lambda, u)$, represents a value of $\lambda$ and a nodal solution $u$ of (1.1) for that particular value of $\lambda$.

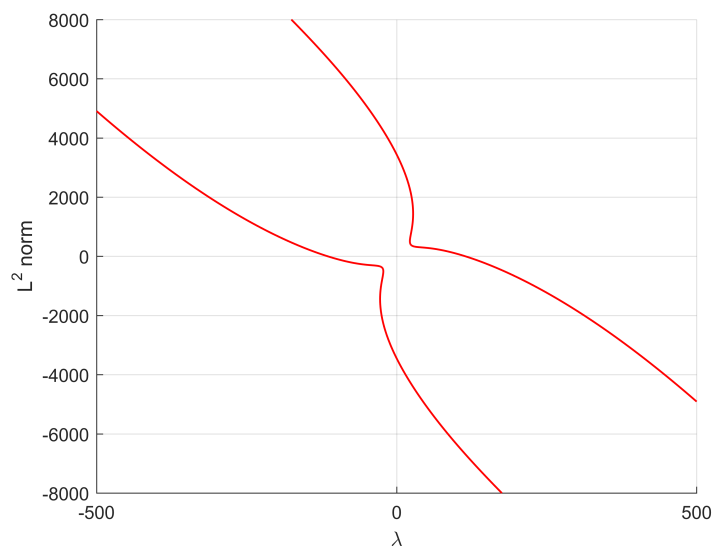

(a) $\mu=35$

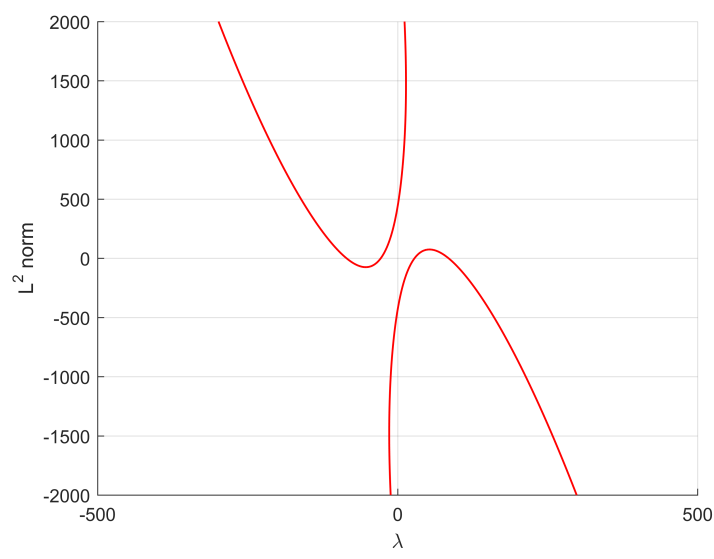

(c) $\mu=45$

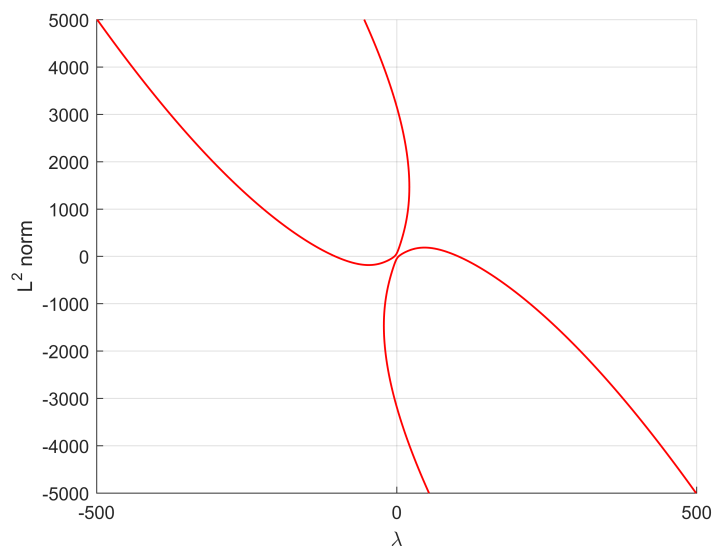

(b) $\mu=39.6$

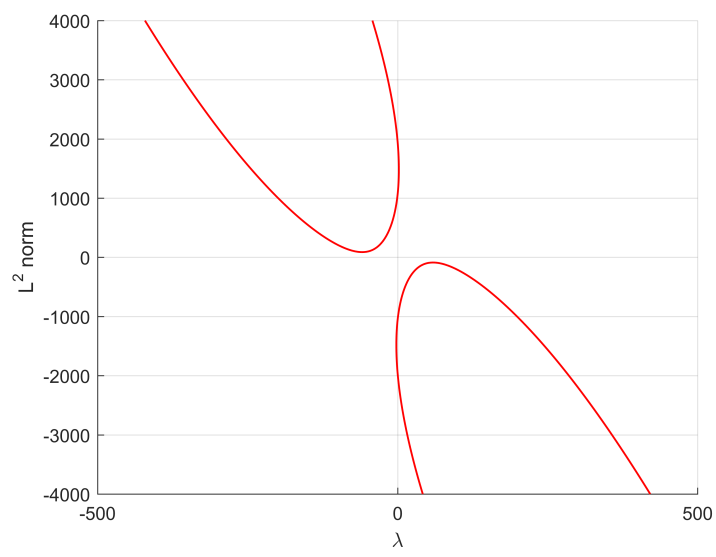

(d) $\mu=54$

FiguRE 5. Four representative bifurcation diagrams of 1-node solutions.

When $\mu$ grows to reach the critical value $(2 \pi)^{2}$, the two components become closer and closer until they meet at $\lambda=0$ at $\mu=(2 \pi)^{2}$, where the set of bifurcation points to 1 -node solutions from $u=0$ consists of the points $\left(\lambda_{ \pm}\left((2 \pi)^{2}\right), 0\right)$ plus $(0,0)$. This is the situation sketched by Figure $5 \mathrm{~b}$, where we have plotted the global bifurcation 
diagram computed for

$$
\mu=39.6>39.4786 \sim(2 \pi)^{2} .
$$

When $\mu \in\left((2 \pi)^{2}, \mu_{2}\right)$, where $\mu_{2}$ is given by (1.7), the set $\Sigma_{2}^{-1}(\mu)$ consists of four values: two negative, $\lambda_{-[1,2]}(\mu)<\lambda_{-[2,2]}(\mu)<0$, plus two positive, $0<\lambda_{+[2,2]}(\mu)<\lambda_{+[1,2]}(\mu)$. Moreover, by Proposition 2.2, it is apparent that

$$
0<\lambda_{+[2,2]}(\mu)=-\lambda_{-[2,2]}(\mu)<\lambda_{+[1,2]}(\mu)=-\lambda_{-[1,2]}(\mu) .
$$

Furthermore, as suggested by our numerical experiments,

$$
\dot{\Sigma}_{2}\left(\lambda_{-[1,2]}(\mu)\right)>0, \quad \dot{\Sigma}_{2}\left(\lambda_{-[2,2]}(\mu)\right)<0, \quad \dot{\Sigma}_{2}\left(\lambda_{+[2,2]}(\mu)\right)>0, \quad \dot{\Sigma}_{2}\left(\lambda_{+[1,2]}(\mu)\right)<0 .
$$

Thus, again the transversality condition of [12] holds at each of these critical values of the parameter $\lambda$. Therefore, (1.1) should possess four analytic curves filled in by 1 -node solutions bifurcating from $u=0$ at each of these critical values of the parameter $\lambda$. Figure $5 \mathrm{c}$ shows the global bifurcation diagram of 1-node solutions bifurcating from these four bifurcation points that we have computed for $\mu=45$. Once again, the set of 1-node solutions consists of two components.

As soon as the transversality condition of Crandall and Rabinowitz [12] holds, the generalized algebraic multiplicity of Esquinas and López-Gómez [15, 26], ₹, equals 1 and hence, thanks to Theorem 5.6.2 of López-Gómez [26], the Leray-Schauder index of $u=0$, as a solution of (1.1), changes as $\lambda$ crosses each of these values. Therefore, each component of the set of non-trivial solutions of (1.1) emanating from $u=0$ at each of these critical values of the primary parameter $\lambda$ satisfies the global alternative of Rabinowitz [41], i.e., either it is unbounded in $\mathbb{R} \times \mathcal{C}[0,1]$, or it meets the trivial solution in, at least, two of these singular values.

Each of the two components plotted in Figure $5 \mathrm{c}$ bifurcates from two different points of $(\lambda, 0)$ and, according to our numerical experiments, both seem to be unbounded. The problem of ascertaining their precise global behavior remains open. As $\mu$ increases and crosses the critical value $\mu_{2}$, these two components disconnect from the trivial solution and stay separated from it, becoming isolas, i.e., components separated from the trivial branch. Figure 5d shows the plots of these components for $\mu=54$. In Figure 6 we have plotted some distinguished solutions with 1-node along some of the pieces of the global bifurcation diagrams of Figure 5. Namely, Figure $6 \mathrm{~b}$ shows a series of solutions with one node along the same-colored branches of Figure 6a, which is a magnification of a part of the left component of Figure 5a, and Figure 6d shows a series of solutions with one node along the bifurcation diagram plotted in Figure 6c, which is a magnification of a part of the left component plotted in Figure 5d. The colors of each of these 1-node solutions correspond to the color of the part of the bifurcation diagram on the left where they are coming from.

Similarly, according to Theorem 2.1, for the special choice (3.1), the third eigencurve, $\Sigma_{3}(\lambda)$, is not concave if (3.1) holds. This can be seen in the plot of $\Sigma_{3}(\lambda)$ in Figure 2. For every $\mu \in\left((3 \pi)^{2}, \mu_{3}\right)$, the set $\Sigma_{3}^{-1}(\mu)$ consists of two negative eigenvalues, $\lambda_{-[1,3]}(\mu)<\lambda_{-[2,3]}(\mu)<0$ and two positive eigenvalues, $0<\lambda_{+[2,3]}(\mu)<\lambda_{+[1,3]}(\mu)$. Moreover, by Proposition 2.2,

$$
0<\lambda_{+[2,3]}(\mu)=-\lambda_{-[2,3]}(\mu)<\lambda_{+[1,3]}(\mu)=-\lambda_{-[1,3]}(\mu)
$$




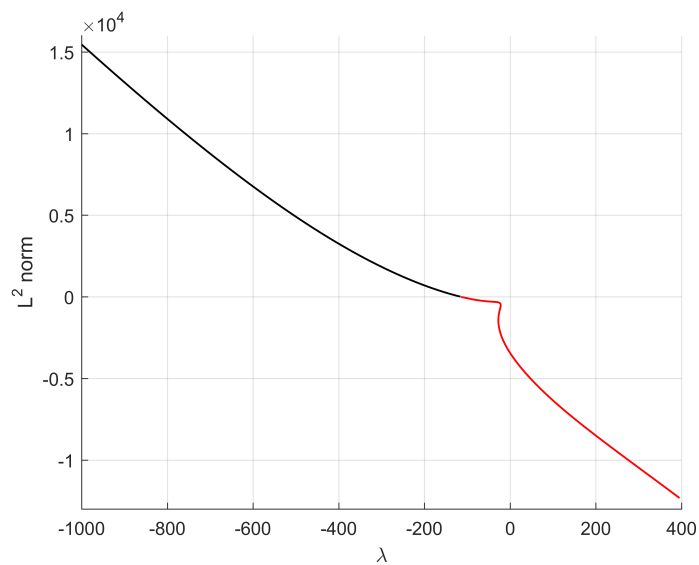

(a) A part of the left branch of Figure $5 \mathrm{a}$ for $\mu=35$

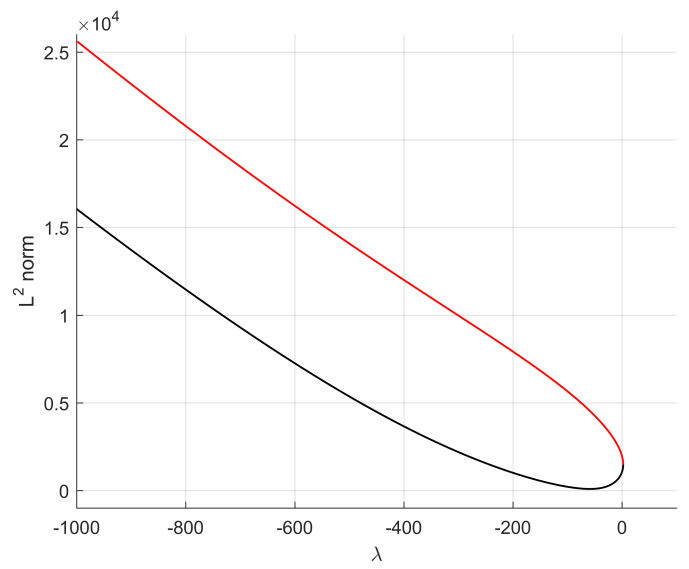

(c) A part of the left branch of Figure $5 \mathrm{~d}$ for $\mu=54$

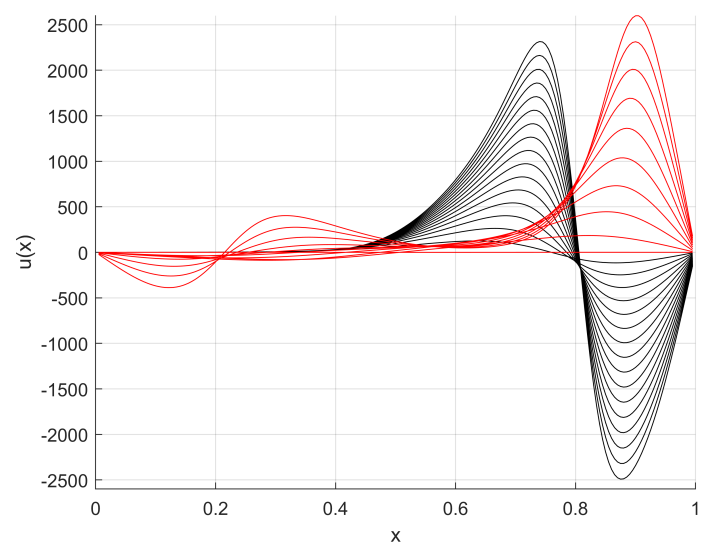

(b) A series of same-colored solutions on the left branches

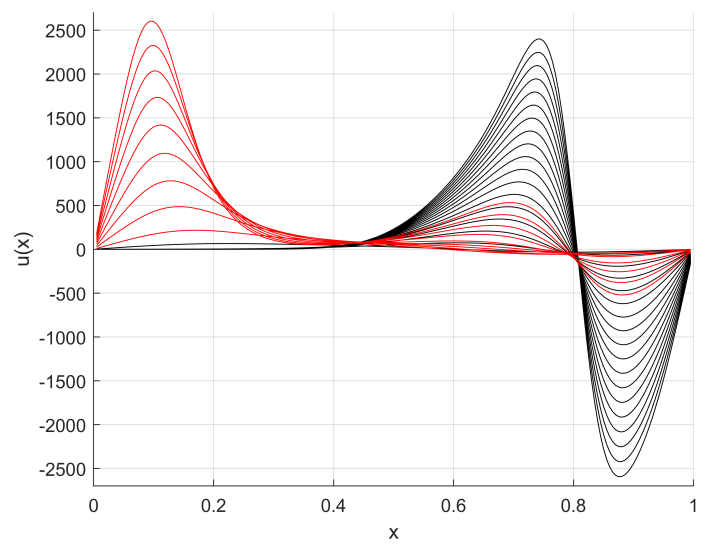

(d) A series of same-colored solutions on the left branches

Figure 6. A series of plots of 1-node solutions (right) along some of the same-colored components of Figure 5 (left).

and, according to our numerical experiments,

$$
\dot{\Sigma}_{2}\left(\lambda_{-[1,3]}(\mu)\right)>0, \quad \dot{\Sigma}_{2}\left(\lambda_{-[2,3]}(\mu)\right)<0, \quad \dot{\Sigma}_{2}\left(\lambda_{+[2,3]}(\mu)\right)>0, \quad \dot{\Sigma}_{2}\left(\lambda_{+[1,3]}(\mu)\right)<0 .
$$

Thus, the transversality condition of [12] holds at each of these critical values and the local bifurcation theorem of [12] implies that an analytic curve of 2-node solutions emanates from $u=0$ at each of these four singular values of $\lambda$. The first three plots of Figure 7 show these curves for three different values of the secondary parameter $\mu$. Namely: $\mu=105, \mu=108.1$ and $\mu=110$, respectively. All these values satisfy $\mu<\mu_{3}$. The last plot of Figure 7 has been computed for $\mu=140>\mu_{3}$ and shows three components of 2-node solutions separated from $u=0$. For this value of $\mu$ no solution with 2 interior nodes can bifurcate from $u=0$.

More precisely, at $\mu=105$ the problem (1.1) possesses three components of solutions with two interior nodes. Two of them bifurcating from $u=0$ at $\lambda_{-[1,3]}(105)$ and 


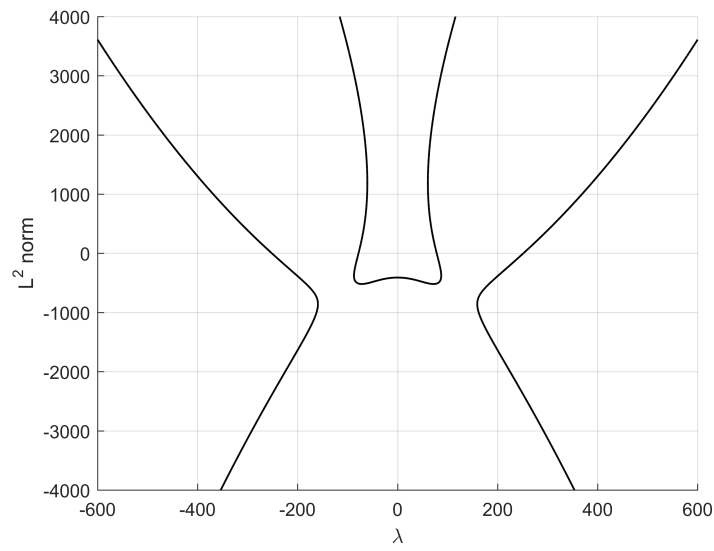

(a) $\mu=105$

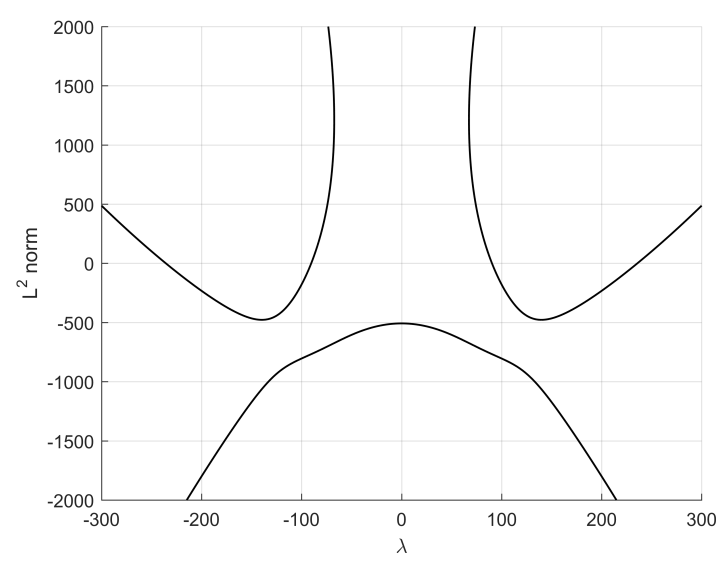

(c) $\mu=110$

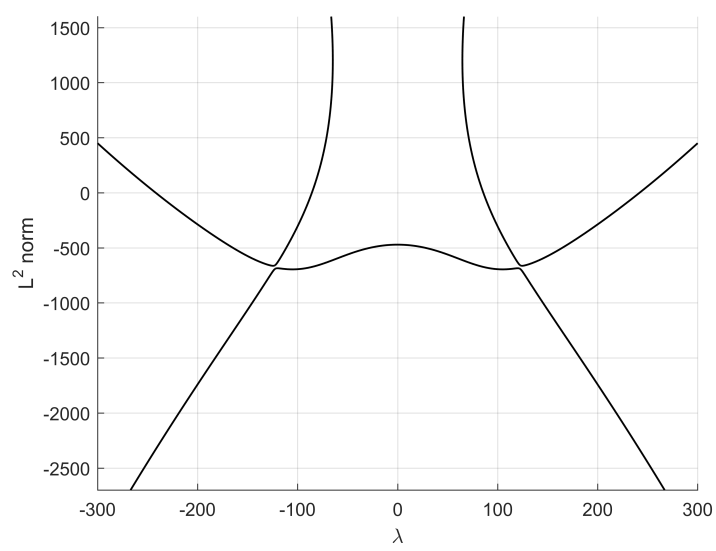

(b) $\mu=108.1$

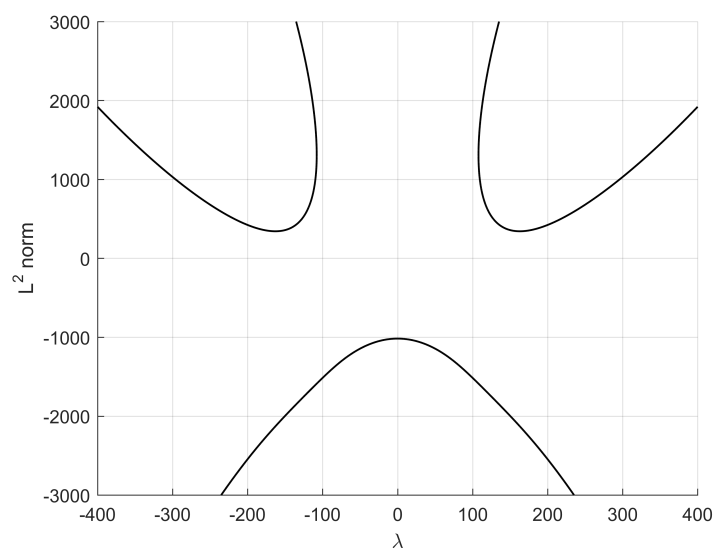

(d) $\mu=140$

FiguRE 7. Four representative bifurcation diagrams of 2-node solutions.

$\lambda_{+[1,3]}(105)$, respectively, and the third one linking $\left(\lambda_{-[2,3]}(105), 0\right)$ with $\left(\lambda_{+[2,3]}(105), 0\right)$. According to our numerical experiments these components are unbounded in $\mathbb{R} \times \mathcal{C}[0,1]$, and are persistent for all further value of $\mu$ below some critical value, $\mu_{c}<108.1$, where the three components meet. Thus, for $\mu=\mu_{c}$ there is a component of the set of nontrivial solutions of (1.1) bifurcating from $u=0$ at four different values of $\lambda: \lambda_{ \pm[1,3]}\left(\mu_{c}\right)$ and $\lambda_{ \pm[2,3]}\left(\mu_{c}\right)$. The plot in Figure $7 \mathrm{~b}$ shows the corresponding global bifurcation diagram for $\mu=108.1$, a value of $\mu$ slightly greater than $\mu_{c}$, where the three components of non-trivial solutions are very close. Comparing to the global bifurcation diagram for $\mu=105$, it becomes apparent that a global imperfect bifurcation has happened at the critical value $\mu_{c}$. As a consequence, one of the components bifurcating from $u=0$ links $\left(\lambda_{-[1,3]}(108.1), 0\right)$ with $\left(\lambda_{-[2,3]}(108.1), 0\right)$, another links $\left(\lambda_{+[2,3]}(108.1), 0\right)$ with $\left(\lambda_{+[1,3]}(108.1), 0\right)$, while the third one remains separated from $u=0$. The latter remains separated from zero for any further value of $\mu$. Therefore, a reorganization of the components of the set of 2-node solutions occurs as $\mu$ crosses $\mu_{c}$. The pictures in Figures $7 \mathrm{c}, 7 \mathrm{~d}$ show the plots of the corresponding components for $\mu=110<\mu_{3}$ 
and $\mu=140>\mu_{3}$, where the previous bifurcations from $u=0$ of these components are lost. For larger values of $\mu$ the solutions along these three components become larger and larger and it remains an open problem to ascertain whether, or not, (1.1) can admit some 2 -node solution for sufficiently large $\mu$. Figure 8 shows the plots of some distinguished 2-node solutions of (1.1) along some of the curves of the bifurcation diagrams plotted in Figure 7.

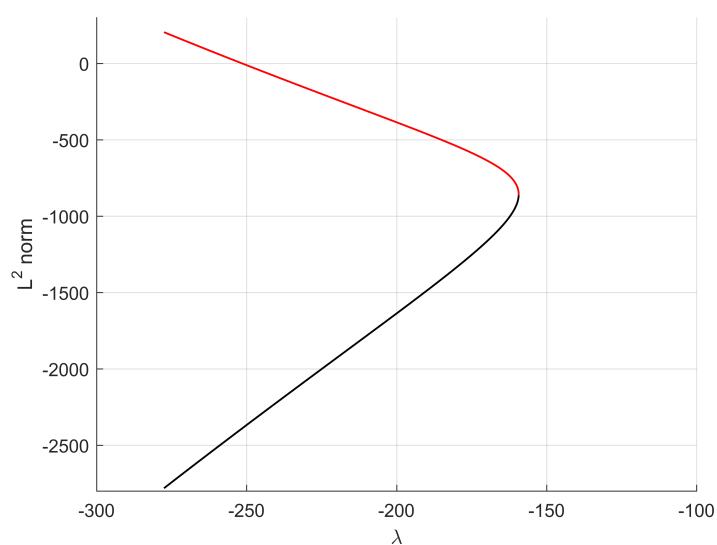

(a) Left branch of Figure 7a $(\mu=105)$

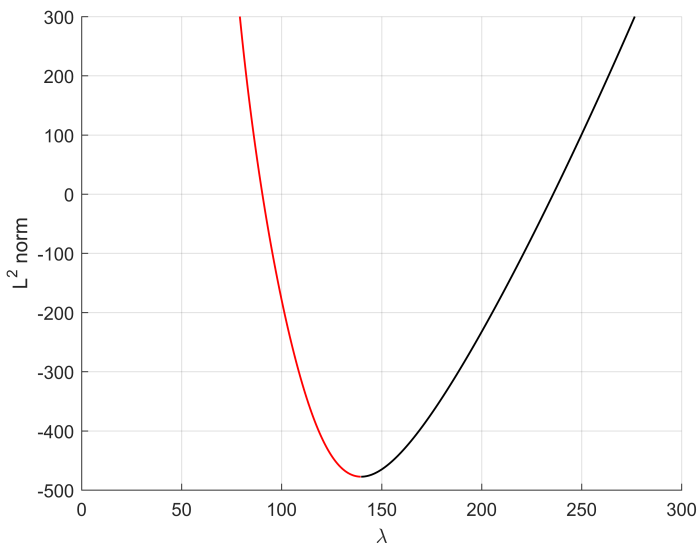

(c) Right branch of Figure 7c $(\mu=110)$

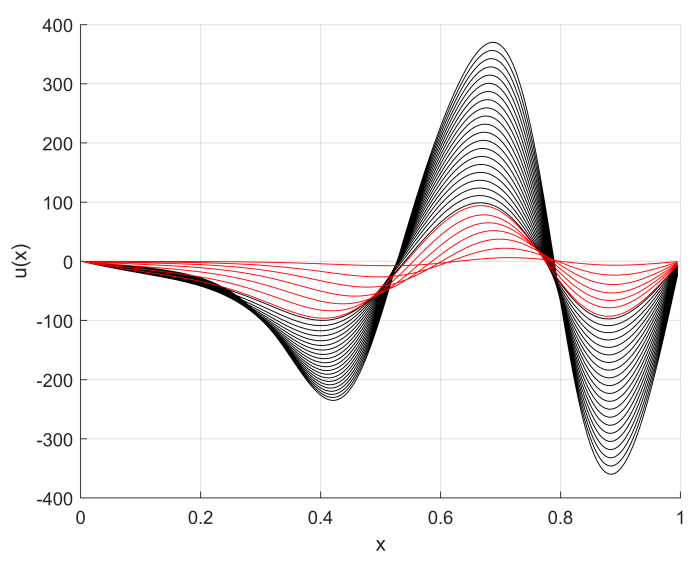

(b) Corresponding profiles of solutions.

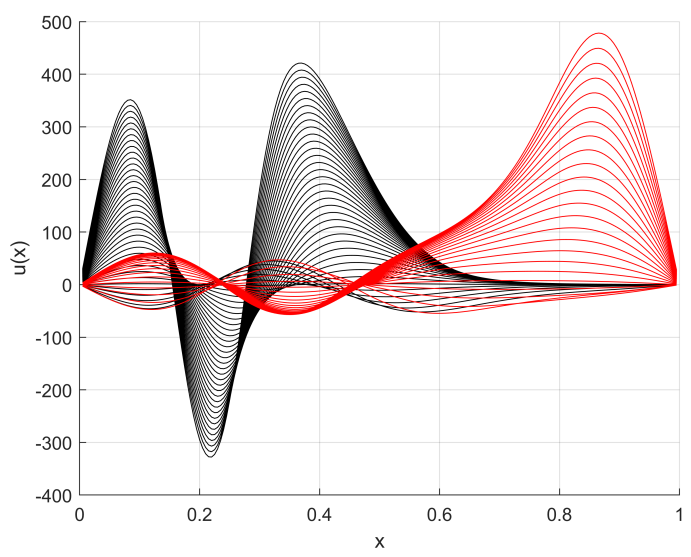

(d) Corresponding profiles of solutions

FIGURE 8. Some plots of 2-node solutions (right) along the bifurcation diagrams of Figure 7 (left).

Finally, Figure 9 contains the global bifurcation diagrams of positive solutions found in [29] (in blue) together with the global bifurcation diagrams of nodal solutions with one node (in red) and two nodes (in black) computed in this paper for four different values of $\mu$ : $0,54,70$ and 100. Although all the components of nodal solutions persist for these values of $\mu$, the component of positive solutions shrinks to a single point and disappears at a value of $\mu$ above 54 but very close to it. In Figure $9 \mathrm{~b}$ one can still see an small piece of blue trace component shortly before disappearing for an slightly greater value of $\mu$. 


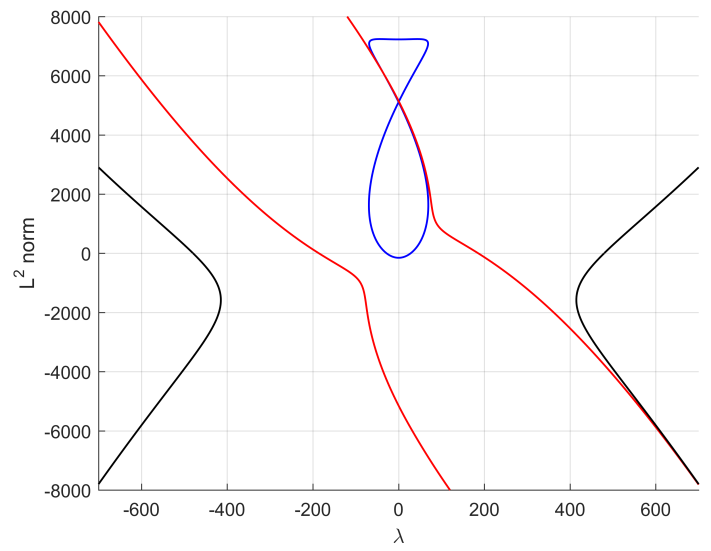

(a) $\mu=0$

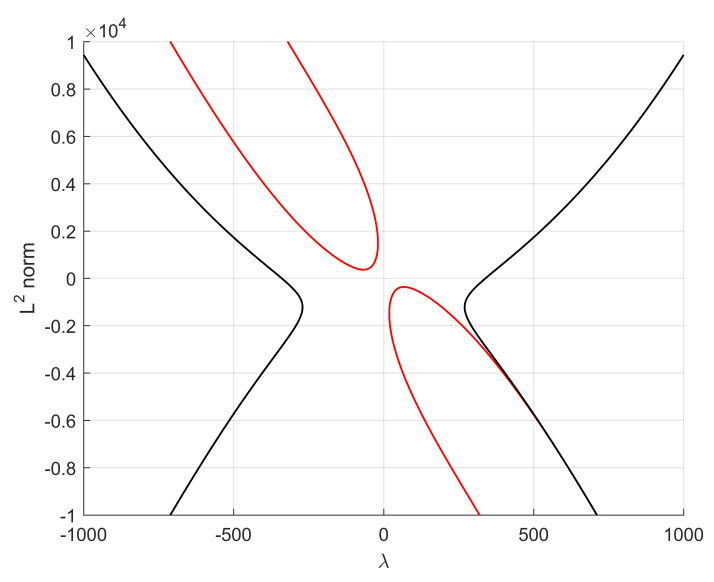

(c) $\mu=70$

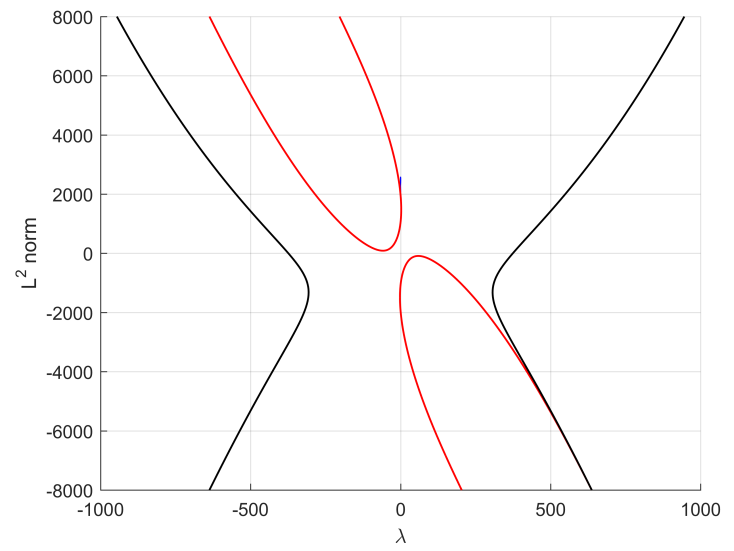

(b) $\mu=54$

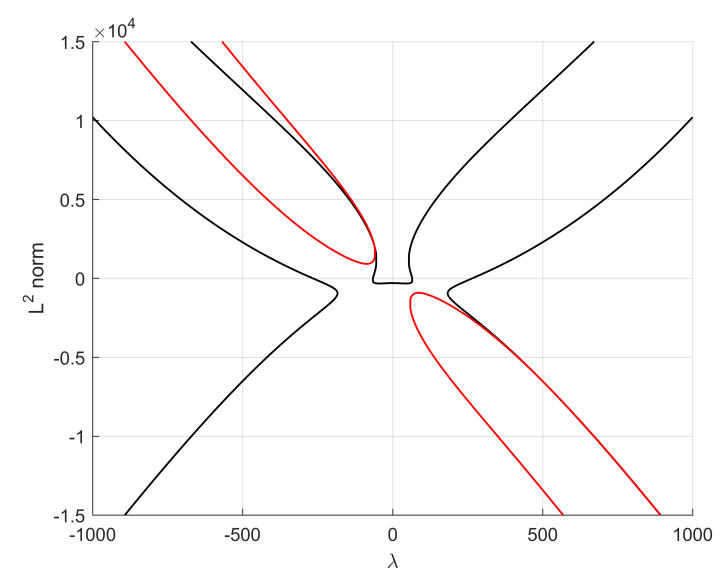

(d) $\mu=100$

FiguRE 9. Some bifurcation diagrams with superimposed branches of positive (blue), 1-node (red) and 2-node (black) solutions.

\section{Numerical METHODS AND THEIR IMPLEMENTATION}

To discretize (1.1) we have used two methods. To compute the small solutions bifurcating from $u=0$ we implemented a pseudo-spectral method combining a trigonometric spectral method with collocation at equidistant points, as in most of the previous numerical experiments of the second author and coworkers (see, e.g., [18, 19, 28, 30, 31, $32,33]$ ). This gives high accuracy (see, e.g., Canuto, Hussaini, Quarteroni and Zang [11]). However, to compute the large solutions we have used a centered finite differences scheme, which gives high accuracy at a lower computational cost. This provides us with a much faster code to compute global solution curves.

The pseudo-spectral method is easier to use and more efficient for choosing the shooting direction from the trivial solution in order to compute the small nodal solutions of (1.1), as well as to detect bifurcation points along the bifurcation diagrams. This is due to the fact that it provides us with the true bifurcation values from $u=0$, while the schemes in differences only approximate them. 
For general Galerkin approximations, the local convergence of the solution paths at regular, turning and simple bifurcation points was proven by Brezzi, Rappaz and Raviart in $[6,7,8]$ and by López-Gómez et al. in [28, 35] at codimension two singularities in the context of systems. In these situations, the local structure of the solution sets for the continuous and the discrete models are known to be equivalent. The global continuation solvers used to compute the solution curves of this papers, as well as the dimensions of the unstable manifolds of all the solutions along them, have been built from the theory on continuation methods of Allgower and Georg [1], Crouzeix and Rappaz [13], Eilbeck [16], Keller [23], López-Gómez [25] and López-Gómez, Eilbeck, Duncan and Molina-Meyer [28].

The complexity of the bifurcation diagrams, as well as their quantitative features, required an extremely careful control of all the steps in subroutines. This explains why the available commercial bifurcation packages, such as AUTO-07P are not useful to deal with differential equations with heterogeneous coefficients. As a matter of fact, Doedel and Oldeman admitted in [14, p.18] that

"Note that, given the non-adaptive spatial discretization, the computational procedure here is not appropriate for PDEs with solutions that rapidly vary in space, and care must be taken to recognize spurious solutions and bifurcations."

This is just one of the main problems that we found in our numerical experiments, as the number of critical points of the solutions increases according to the dimensions of unstable manifolds, and the turning and bifurcation points might be very close.

Acknowledgements. We thank the anonymous reviewer for his/her extremely careful reading of the paper, which has greatly improved it.

\section{REFERENCES}

[1] E. L. Allgower and K. Georg, Introduction to Numerical Continuation Methods, SIAM Classics in Applied Mathematics 45, SIAM, Philadelphia, 2003.

[2] I. Antón and J. López-Gómez, Principal eigenvalues of weighted periodic-parabolic problems, Rend. Istit. Mat. Univ. Trieste 49 (2017), 287-318.

[3] W. Arendt, C. J. K. Batty, M. Hieber and F. Neubrander, Vector-valued Laplace transforms and Cauchy problems, Monographs in Mathematics vol. 96, Birkhäuser/Springer, Basel, 2011.

[4] W. Arendt, A. Grabosch, G. Greiner, U. Groh, H. P. Lotz, U. Moustakas, R. Nagel, F. Neubrander and U. Schlotterbeck, One Parameter Semigroups of Positive Operators, Lectures Notes in Mathematics 1184, Berlin, Springer, 1986.

[5] H. Berestycki, L. Nirenberg and S. R. S. Varadhan, The principal eigenvalue and maximum principle for second order elliptic operator sin general domains, Comm. Pure Appl. Math. 47 (1994), 47-92.

[6] F. Brezzi, J. Rappaz and P. A. Raviart, Finite dimensional approximation of nonlinear problems, part I: Branches of nonsingular solutions, Numer. Math. 36 (1980), 1-25.

[7] F. Brezzi, J. Rappaz and P. A. Raviart, Finite dimensional approximation of nonlinear problems, part II: Limit points, Numer. Math. 37 (1981), 1-28.

[8] F. Brezzi, J. Rappaz and P. A. Raviart, Finite dimensional approximation of nonlinear problems, part III: Simple bifurcation points, Numer. Math. 38 (1981), 1-30.

[9] G. Buttazzo, M. Giaquinta and S. Hildebrandt, One-dimensional Variational Problems, Clarendon Press, Oxford, 1998. 
[10] S. Cano-Casanova and J. López-Gómez, Properties of the principal eigenvalues of a general class of nonclassical mixed boundary value problems, J. Dif. Eqns. 178 (2002), 123-211.

[11] C. Canuto, M. Y. Hussaini, A. Quarteroni and T. A. Zang, Spectral Methods in Fluid Mechanics, Springer, Berlin, Germany, 1988.

[12] M. G. Crandall and P. H. Rabinowitz, Bifurcation from simple eigenvalues, J. Funct. Anal. 8 (1971), 321-340.

[13] M. Crouzeix and J. Rappaz, On Numerical Approximation in Bifurcation Theory, Recherches en Mathmatiques Appliques 13, Masson, Paris, 1990.

[14] E. J. Doedel and B. E. Oldeman, AUTO-07P: Continuation and bifurcation software for ODEs, 2012, http://www.dam.brown.edu/people/sandsted/auto/auto07p.pdf.

[15] J. Esquinas and J. López-Gómez, Optimal multiplicity in local bifurcation theory: Generalized generic eigenvalues, J. Diff. Eqns. 71 (1988), 72-92.

[16] J. C. Eilbeck, The pseudo-spectral method and path-following in reaction-diffusion bifurcation studies, SIAM J. Sci. Stat. Comput. 7 (1986), 599-610.

[17] D. G. de Figueiredo, Positive Solutions of Semilinear Elliptic Problems, Lectures Notes of a LatinAmerican School on Differential Equations, Sao Paolo 1981, Lectures Notes in Mathematics 957 (pp. 34-87), Springer, 1982.

[18] R. Gómez-Reñasco and J. López-Gómez, The effect of varying coefficients on the dynamics of a class of superlinear indefinite reaction diffusion equations, J. Diff. Eqns. 167 (2000), 36-72.

[19] R. Gómez-Reñasco and J. López-Gómez, On the existence and numerical computation of classical and non-classical solutions for a family of elliptic boundary value problems, Nonl. Anal. TMA 48 (2002), 567-605.

[20] P. Hess and T. Kato, On some linear and nonlinear eigenvalue problems with an indefinite weight function, Comm. Part. Dif. Eqns. 5 (1980), 999-1030.

[21] T. Kato, Superconvexity of the spectral radius and convexity of the spectral bound and the type, Math. Z. 180 (1982), 265-273.

[22] T. Kato, Perturbation Theory for Linear Operators, Springer, 1995.

[23] H. B. Keller, Lectures on Numerical Methods in Bifurcation Problems, Tata Insitute of Fundamental Research, Springer, Berlin, Germany, 1986.

[24] H. B. Keller and Z. H. Yang, A direct method for computing higher order folds, SIAM J. Sci. Stat. 7 (1986), 351-361.

[25] J. López-Gómez Estabilidad y Bifurcación Estática. Aplicaciones y Métodos Numéericos, Cuadernos de Matemática y Mecánica, Serie Cursos y Seminarios 4, Santa Fe, R. Argentina, 1988.

[26] J. López-Gómez, Spectral Theory and Nonlinear Functional Analysis, CRC Press, Boca Raton, 2001.

[27] J. López-Gómez, Linear Second Order Elliptic Operators, World Scientific Publishing, 2013.

[28] J. López-Gómez, J. C. Eilbeck, K. Duncan and M. Molina-Meyer, Structure of solution manifolds in a strongly coupled elliptic system, IMA J. Numer. Anal. 12 (1992), 405-428.

[29] J. López-Gómez and M. Molina-Meyer, Bounded components of positive solutions of abstract fized point equations: mushrooms, loops and isolas, J. Diff. Eqns. 209 (2005), 416-441.

[30] J. López-Gómez and M. Molina-Meyer, Superlinear indefinite systems: Beyond Lotka Volterra models, J. Differ. Eqns. 221 (2006), 343-411.

[31] J. López-Gómez and M. Molina-Meyer, The competitive exclusion principle versus biodiversity through segregation and further adaptation to spatial heterogeneities, Theor. Popul. Biol. 69 (2006), 94-109.

[32] J. López-Gómez and M. Molina-Meyer, Modeling coopetition, Math. Comput. Simul. 76 (2007), $132-140$.

[33] J. López-Gómez, M. Molina-Meyer and A. Tellini, Intricate dynamics caused by facilitation in competitive environments within polluted habitat patches, Eur. J. Appl. Maths. doi:10.1017/S0956792513000429 (2014), 1-17. 
[34] J. López-Gómez, M. Molina-Meyer and P. H. Rabinowitz, Global bifurcation diagrams of one node solutions in a class of degenerate bundary value problems, Disc. Cont. Dyn. Sys. B 22 (2017), 923-946.

[35] J. López-Gómez, M. Molina-Meyer and M. Villareal, Numerical coexistence of coexistence states, SIAM J. Numer. Anal. 29 (1992), 1074-1092.

[36] J. López-Gómez, A. Tellini and F. Zanolin, High multiplicity and complexity of the bifurcation diagrams of large solutions for a class of superlinear indefinite problems, Commun. Pure Appl. Anal. 13 (2014), 1-73.

[37] J. López-Gómez and P. H. Rabinowitz, Nodal solutions for a class of degenerate boundary value problems, Adv. Nonl. Studies 15 (2015), 253-288.

[38] J. López-Gómez and P. H. Rabinowitz, Nodal solutions for a class of degenerate one dimensional BVPs, Top. Meth. Nonl. Anal. 49 (2017), 359-376.

[39] J. López-Gómez and P. H. Rabinowitz, The structure of the set of 1-node solutions of a class of degenerate BVP's, J. Diff. Eqns. 268 (2020), 4691-4732.

[40] J. Mawhin, D. Papini and F. Zanolin, Boundary blow-up for differential equations with indefinite weight, J. Diff. Eqns. 188 (2003), 33-51.

[41] P. H. Rabinowitz, Some global results for nonlinear eigenvalue problems, J. Funct. Anal. 7 (1971), 487-513.

[42] P. H. Rabinowitz, A note on a nonlinear eigenvalue problem for a class of differential equations, J. Diff. Eqns. 9 (1971), 536-548.

[43] P. H. Rabinowitz, A note on a pair of solutions of a nonlinear Sturm-Liouville problem, Manuscr. Math. 11 (1974), 273-282.

Department of Mathematics and NTIS, Faculty of Applied Sciences, University of West Bohemia, Univerzitní 8, 30100, Plzen, Czech Republic

E-mail address: fenclm37@ntis.zcu.cz

Institute of Inter-Disciplinar Mathematics (IMI) And Department of AnAlysis And Applied Mathematics, Complutense University of Madrid, Madrid 28040, Spain

E-mail address: Lopez_Gomez@mat.ucm.es 